\title{
Integration of a testbench for the optical and thermal characterization of near-infrared detectors used in ground and space-based astronomy
}

\author{
Jorge Jiménez, Antoni Grau, Senior Member, IEEE and Cristobal Padilla
}

\begin{abstract}
The mercury-cadmium-tellurium (MCT) sensors are considered the ideal infrared detectors to equip the focal plane arrays (FPAs) of astronomical scientific missions and nowadays, Europe is not self-sufficient on this technology. To tackle this problem, the Astronomy European Infrared Detector (ASTEROID) project aims to provide Europe with the capability to manufacture high performance infrared focal plane arrays devoted to scientific use, space astronomy and ground-based telescope infrastructures. The resulting detector will be a hybridized MCT array with a CdZnTe substrate of $2 \mathrm{k} \times 2 \mathrm{k}$ pixels and $15 \mu \mathrm{m}$ of pixel pitch. The Institut de Física d'Altes Energies (IFAE) will lead the task to validate the detector performance with respect to the hybridization reliability between the readout integrated circuit (ROIC) and the $M C T$ detector by submitting it to several low temperature thermal cycles. After every thermal stress, the detector will be optically characterized in order to measure any performance degradation and possible problems with the pixel operability. To perform such tasks, two custom setups, focused on optical and cryo-vacuum aspects were developed, which used in conjunction, allow to cool down the detector from the room temperature up to $\sim 30 \mathrm{~K}$ while it is electro-optically characterized in terms of linearity, gain, quantum efficiency and cosmetics defects, among others. The whole cryo-vacuum system and the optical setup is fully described in this paper as part of the work packages assigned to IFAE to test the hybridized detector to assess the reliability at operating temperature and after several thermal cycles.
\end{abstract}

Index Terms-infrared detectors, characterization setup, vacuum dewar, temperature control

This work was supported by the European Commission under the program H2020-COMPET-2016, grants H2020-EU.2.1.6.2 and H2020-EU.2.1.6.1.1 and the Spanish Science Funding Agency MINECO under grants ESP2015-66861-C3-3-R and ESP201789838-C3-2-R.

Jorge Jiménez is with the Institut de Física d'Altes Energies (IFAE), Campus UAB, 08193 Barcelona, Spain (e-mail: jjimenez@ifae.es).

Antoni Grau is with the Automatic Control Dept, Universitat Politècnica de Catalunya (UPC), 08034 Barcelona, Spain (e-mail: antoni.grau@upc.edu).

Cristobal Padilla is with the Institut de Física d'Altes Energies (IFAE), Campus UAB, 08193 Barcelona, Spain (e-mail: padilla@ifae.es).
TABLE I

MAIN ALFA DETECTOR CHARACTERISTICS [6]

\begin{tabular}{|l|l|}
\hline Parameter & Value \\
\hline Operating Wavelength & $0.8-2.1 \mu \mathrm{m}$ \\
Cutoff Wavelength & $2.1 \mu \mathrm{m}$ \\
Quantum Efficiency & $74 \%$ mean value \\
Operation Temperature & $100 \mathrm{~K}$ \\
Dark Current @ 100K & $<0.5 e^{-} / \mathrm{sec}$ \\
Linear Well Capacity & $60 k e^{-}$ \\
Non-Linearity & $3.2 \%$ and $2.5 \%$ \\
Cross-Talk (IPC) & $0.6 \%$ to $1.1 \%$ \\
Readout Noise (single CDS) & 11.4 to $11.5 e^{-}$ \\
Readout Speed & $100 \mathrm{kHz}$ \\
\hline
\end{tabular}

\section{INTRODUCTION}

$\mathbf{F}$ EW years ago, the European Space Agency (ESA) promoted the research around the development of large size detectors available for scientific missions. Taking advantage of their long expertise on this field [1] [2], two companies, CEA [3] and Lynred [4], developed the Astronomy Large Format Array detector (ALFA) which was addressed to the ESA's Near Infrared Large Format Sensor Array (NIRLFSA) roadmap for the development of large format focal plane arrays for low fluxes and low noise. The ALFA readout integrated circuit (ROIC), one of the key developments of this novel detector, is composed by a matrix of $2048 \times 2048$ pixels with $15 \mu \mathrm{m}$ pitch and its overall size is close to $30 \times 30 \mathrm{~mm}$. In order to achieve these dimensions, the stitching technique has been implemented in the foundry and the ROIC is able to drive the pixels data through a maximum of 32 outputs channels with a readout speed up to $6 \mathrm{MHz}$, with the options to also readout at $100 \mathrm{kHz}$ with 1 and 4 outputs. Table I summarizes the main ALFA detector performance measured at the CEA-IRFU [5] facilities [6].

Meanwhile, ESA-ALFA program aims to develop the first 2048 x $204815 \mu \mathrm{m}$ pitch detector made in Europe, 
the Astronomy European Infrared Detector (ASTEROID) [7] detector, founded by the European Commission in the frame of a H2020-COMPET program strategy and lead by key research institutions (CEA-Leti [8], CEA$I R F U$ and IFAE [9]) and industrial partners (Lynred, $E V G$ [10] and $A D D L$ [11]). The final goal is to achieve a European IR detector manufacturer ready to produce this $N I R / S W I R$ detector with the requested grade of technology readiness level (TRL). Additionally, ASTEROID will prepare the future technologies ready to mass produce this detector and also to access to larger format detectors such as $4 \mathrm{k} \mathrm{x} 4 \mathrm{k}$.

In order to test the resulting devices, the IFAE is leading the research to validate the detector performance concerning the hybridization reliability between the ROIC and the MCT detector, which is a key process on the way to get better and cost effective focal plane arrays (FPAs). The validation will be performed by submitting the detector to several low temperature thermal cycles in the range between the room temperature and $\sim 50 \mathrm{~K}$. During the thermal cycle tests, the detector will be optically characterized in order to measure any performance degradation, the pixel operability, disconnected or dead, which have special interest for those teams looking to validate the thermo-mechanical model ruling these FPAs.

To carry out such tests, custom setups are demanded, many of them spread along Europe [12] and US [13] with the specifications focused mainly on the electro-optical $(E O)$ characterization of novel detectors or devoted to perform the detector's characterization and validation prior its use on further instrumentation development. All the existing setups have demonstrated that fulfill the requirements to perform the standard $E O$ tests [14] [15] (dark current, relative and absolute quantum efficiency, conversion and electronic gain, CCD charge transfer efficiency, crosstalk and inter-pixel capacitance, linearity and well depth, image persistence, etc) without focusing on the thermal stress issues. This way, two setups were developed at IFAE which fulfill with these stakeholders needs, regarding the suitable optical illumination of the detector by means of a custom optical setup and the appropriate thermal stress using a cryo-vacuum system with capabilities to reach cryogenics thermal cycles. Each setup comes from a different engineering field and interacts between them in a synchronized way. Figure 1 shows a diagram of how the setups are connected to form an integrated system.

\section{DETECTOR DESCRIPTION}

The detectors used in scientific space and ground application, must fulfill with the highest specifications regarding their performance, specially those oriented to

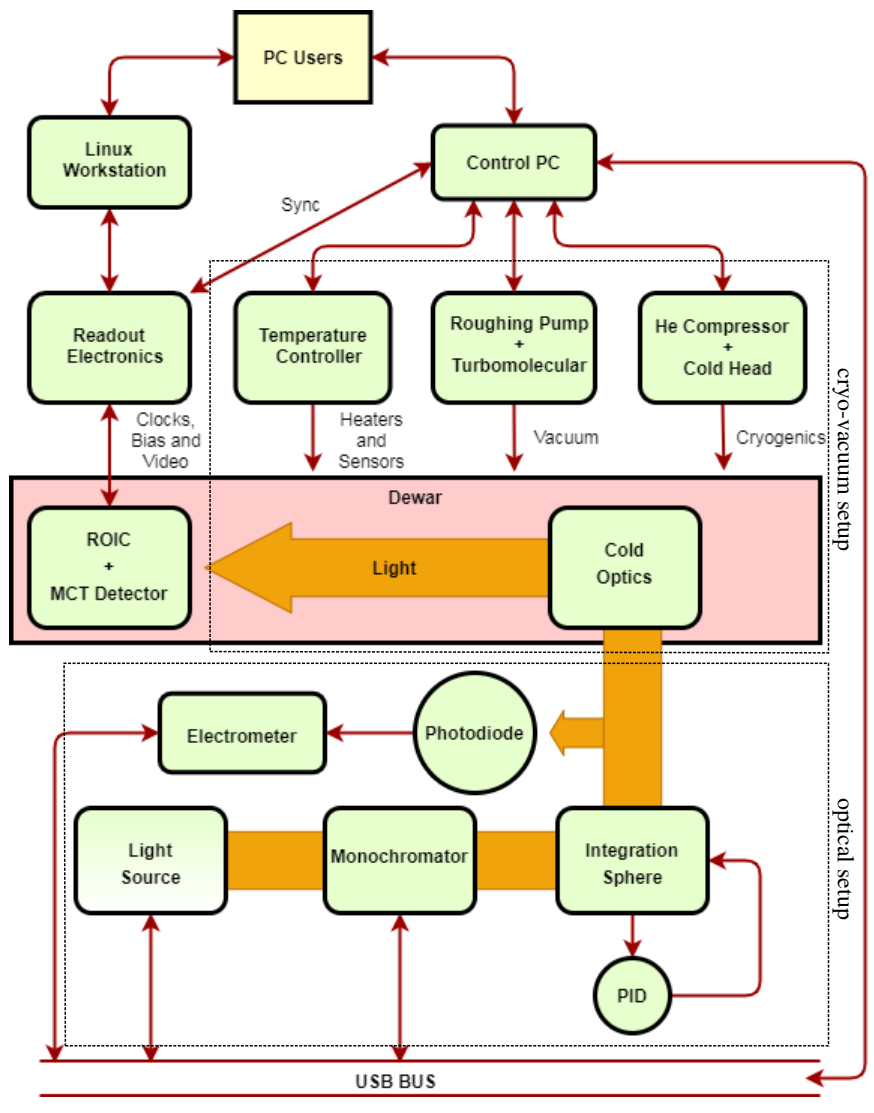

Fig. 1. Block diagram of the integrated setups to perform the characterization of detectors focusing on the thermal stress.

astronomy, where the detector have to meet high $T R L$ levels and support radiation events. For this reason, $M C T$ detectors have been called the ideal infrared detector material, since they offer the higher performance for space application with today's available technology [16]. Moreover, applications on the astronomy domain also impose to deal with two major challenges: i) low photon fluxes; and ii) detectors resolution. The latter is overcome by the manufacturers through the rise of the number of pixels on large FPAs. The low photon fluxes, due to the fact that far away celestial objects are observed or because of the use of narrow-band spectral techniques, is solved using a two-stage approach on the $M C T$ material: firstly, increasing the absorption coefficient or quantum efficiency at the covered detector wavelength range; and secondly, with the detector working at a temperature where the photons due the background temperature equals the dark current, point called background-limited performance (BLIP) [17]. At the end, all these improvements look for not to lose any valuable photon.

As shown in figure 2, dark current strongly decreases by cooling down the detector, which can be predicted by the dark current diffusion model on $M C T$ materials [18]. A rule of thumb for the maximum operation temperature 


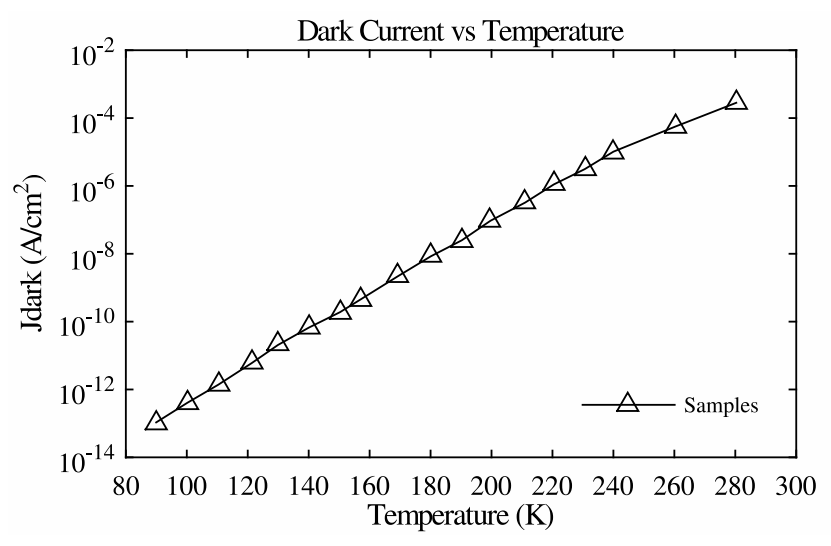

Fig. 2. Dark current vs temperature for the NIRLFSA devices with a cutoff wavelength of $2.45 \mu \mathrm{m}$ [2].

$T_{\max }$ for a detector with a cutoff wavelength $\lambda_{c}$ is [19]:

$$
T_{\max (K)}=\frac{200 K}{\lambda_{c_{(\mu m)}}}
$$

Using this equation, for a detector with a cutoff wavelength of $2.1 \mu \mathrm{m}$, the maximum operation temperature should be $\sim 95 \mathrm{~K}$. In addition to the diffusion dark current which is temperature dependent, there are other ways how the dark current is created but they are mainly related with the manufacturing process and the applied bias voltages.

Quantum efficiency is also temperature dependent since the combination between mercury, cadmium and tellurium in the proportion of $H g_{1-x} C d_{x} T e$ defines the bandgap energy $(\mathrm{eV})$ for the desirable cutoff wavelength. For smaller bandgaps and longer cutoff wavelengths, the temperature modifies the manufacturer's cutoff, which should be considered during the foundry process. As shown in figure 3 , the quantum efficiency depends of the wavelength since the discrete absorption of photons at different wavelengths is performed at diverse depths on the $M C T$ material.

\section{OPTICAL SETUP DESCRIPTION}

To perform the characterization of the ASTEROID detector, an optical setup has been developed at the IFAE optical laboratory [20] [21] with the capability to measure the main performance parameters of a detector: the dark current, linearity, full well, readout noise, quantum efficiency and pixels operability. This custom setup, which optical path is represented in figure 4 , is composed of a Quartz-Tungsten-Halogen $(Q T H)$ lamp source which feeds a $F \# 4$ Czerny-Turner monochromator with its slits adjusted to yield a bandwidth of $10 \mathrm{~nm}$. The monochromatic light is generated through two grating elements with a blaze wavelength at 1200 and $2000 \mathrm{~nm}$,

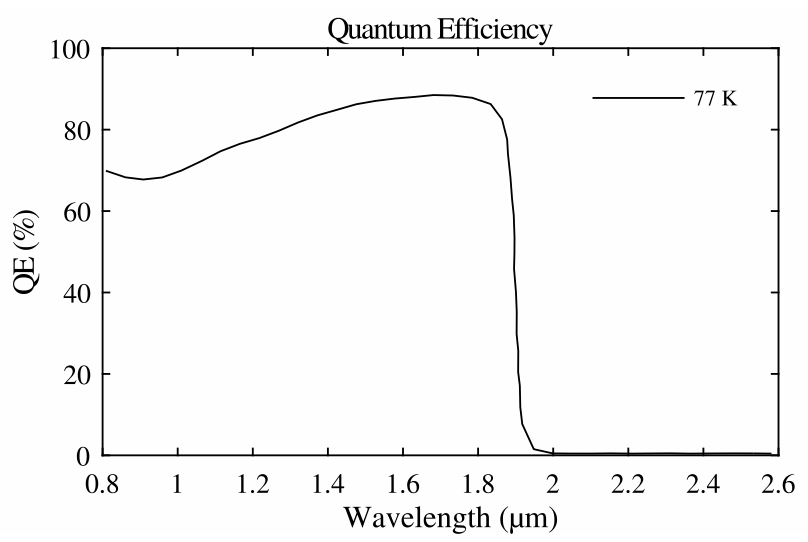

Fig. 3. Typical quantum efficiency for a NIRLFSA device measured on individual diodes at $77 \mathrm{~K}$ [2].

both with a groove density of $300 \mathrm{~g} / \mathrm{mm}$ providing a total dispersion of $19 \mathrm{~nm} / \mathrm{mm}$. A blade shutter and a 6slot filter wheel are located at the entrance port of the monochromator. The filter wheel contains a set of order sorting filters which avoids the second order diffraction at the monochromator output.

The integration sphere is equipped with 3 ports: $0^{\circ}$, $90^{\circ}$ and North-Pole, where the last port holds an InGaAs photodiode used as reference for the irradiance fluctuations. As shown in figure 7, the integration sphere resides inside an aluminium box used as radiation shield. The box and the complete optical path are filled with nitrogen gas that is used to displace the atmospheric air to avoid water absorption in the NIR/SWIR region, which attenuate the $\mathrm{J}, \mathrm{H}$ and $\mathrm{K}$ astronomy bands.

The integration sphere temperature is controlled by a closed-loop PID controller up to reach a relative error of $\sigma \leq 0.008{ }^{\circ} \mathrm{C}$ as shown in figure 5. Such a temperature stability reduces the thermal radiation drift coming from its shell as result of a "black body" behavior, despite the very low emissivity of the gold-coating. As figure 6 depicts, a temperature error of $0.008{ }^{\circ} \mathrm{C}$ in the wave-

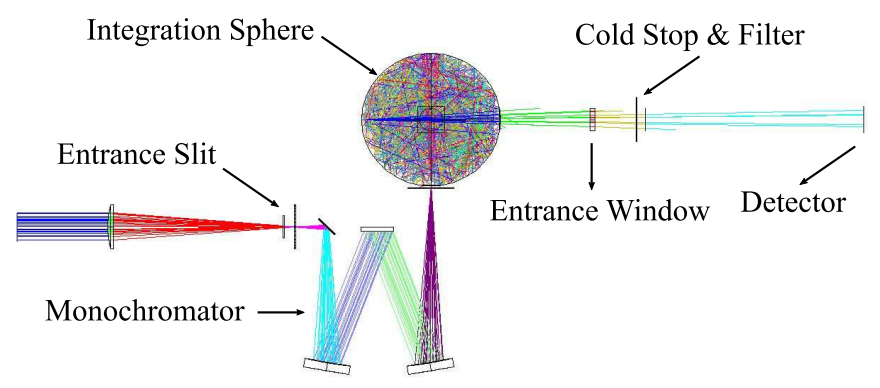

Fig. 4. Optical path layout showing the main parts of the optical setup and the inner cold parts behind the entrance window. 


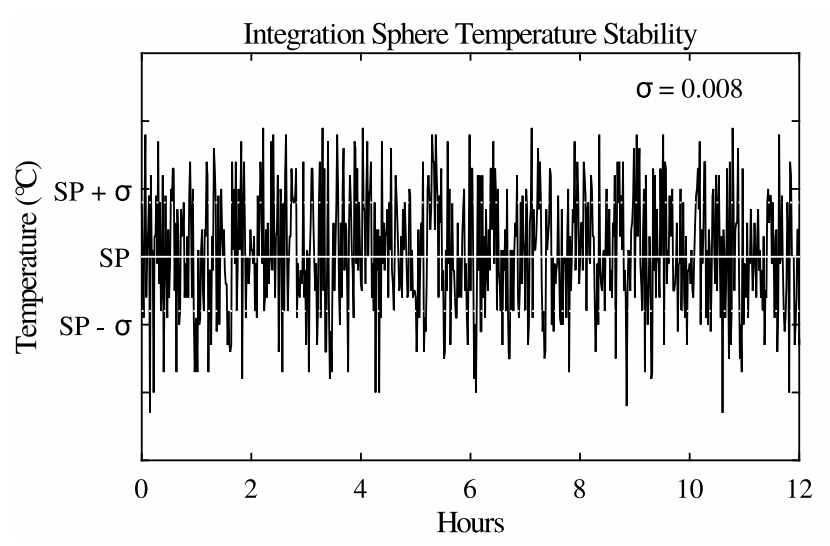

Fig. 5. The temperature of the integration sphere is controlled in order to keep constant the thermal radiation. A closed-loop PID controller allows to reach a setpoint error of $0.008{ }^{\circ} \mathrm{C}$.

length band from 0.8 to $2.5 \mu \mathrm{m}$ corresponds to a total band radiance background of $8.2 e^{-9} \mathrm{~W} / \mathrm{m}^{2}$ and from 0.8 to $1.8 \mu \mathrm{m}$ of $6.8 e^{-12} \mathrm{~W} / \mathrm{m}^{2}$. In order to reduce as much as possible the error while measuring the detectors quantum efficiency, the band corresponding to a range from 0.8 to $1.8 \mu \mathrm{m}$ ( $\mathrm{J}$ and $\mathrm{H}$ astronomy bands) offers the lowest uncertainty and remains below the Noise Equivalent Power (NEP) of any photodiode used in the setup.

In the whole range from 0.8 to $1.8 \mu \mathrm{m}$, the total setup $N E P, N E P_{\text {total }}$, can be approximated by adding in quadrature the NEP from the NIST calibrated photodiode ( $\left.\mathrm{NEP}_{\mathrm{NIST}} \mathrm{PD}\right)$, the InGaAs reference photodiode $\left(\mathrm{NEP}_{\mathrm{REF} P D}\right)$ and the $N E P$ of the integration sphere $\left(\mathrm{NEP}_{\mathrm{IS}}\right)$ as follows:

$$
N E P_{\text {total }}=\sqrt{\mathrm{NEP}_{\mathrm{NIST} \mathrm{PD}^{2}}{ }^{2} \mathrm{NEP}_{\mathrm{REF} \mathrm{PD}^{2}}+\mathrm{NEP}_{\mathrm{IS}}{ }^{2}}
$$

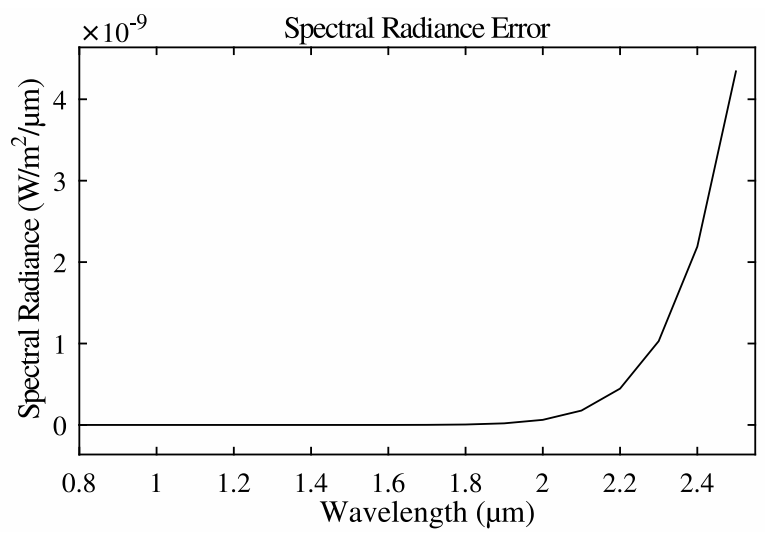

Fig. 6. The low temperature error in the integration sphere $(\sigma \leq$ $0.008{ }^{\circ} \mathrm{C}$ ) produces an uncertainty in the thermal radiation. As any black body source, the spectral radiance is increased at higher wavelengths, specially beyond $2 \mu \mathrm{m}$.

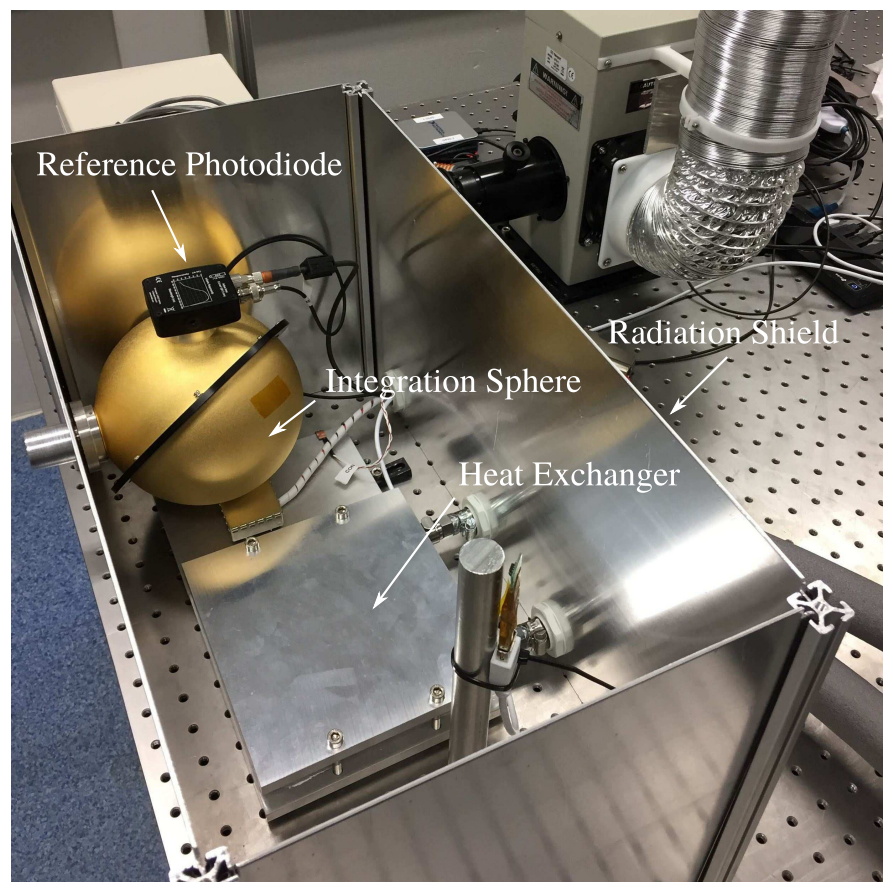

Fig. 7. Inside the aluminium box used as radiation shield, the integration sphere and its heat exchanger stage in a nitrogen gas atmosphere are located.

Specifically, for the proposed setup the value computed is $N E P_{\text {total }}<1.6 e^{-12} \mathrm{~W} / \sqrt{(\mathrm{Hz})}$.

The output of the integration sphere is directly attached to the $\mathrm{CaF}_{2}$ entrance window located at the cryostat. Once inside the cryostat, just behind the entrance window, the cold stop and the cold baffle are located, as shown in figure 8 . Both are aluminium black anodized [22] standard lens tubes: the cold baffle is composed of two $150 \mathrm{~mm}$ long SM2-threaded lens tubes and the cold stop is a $10 \mathrm{~mm}$ SM1-threaded lens tube mounted in the SM2 by means of an adapter. The cold stop size is defined at the first instance by the SM1 lens tube aperture ( $22.9 \mathrm{~mm}$ of clear aperture) but it is finally defined by the opto-mechanics components mounted, i.e. a pinhole and/or a filter.

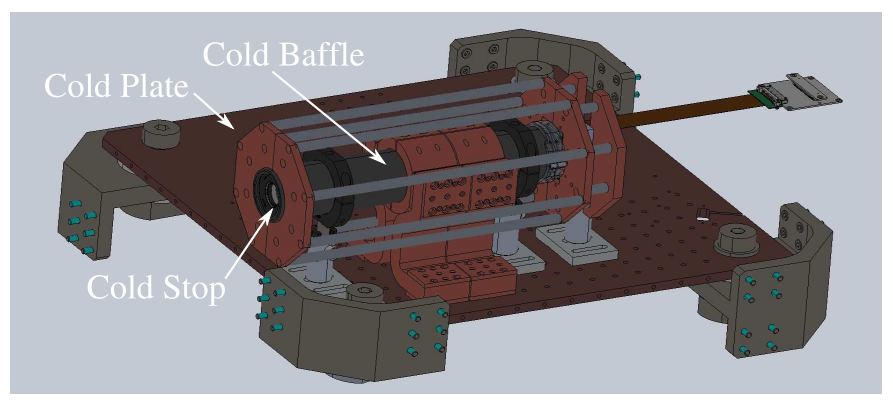

Fig. 8. Inner view of the cryostat where the cold baffle and the cold plate can be appreciated. 


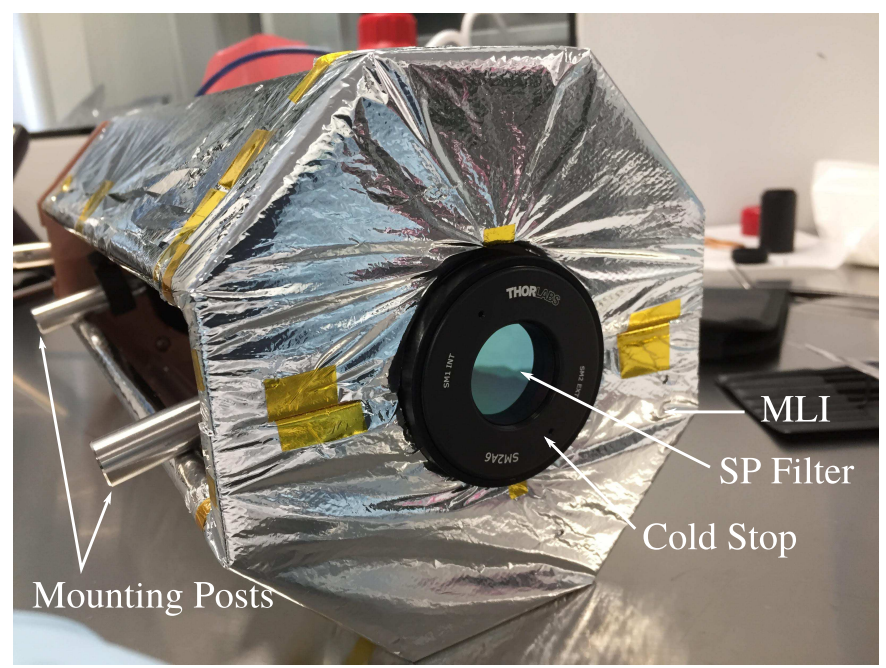

Fig. 9. The whole cold baffle is wrapped with MLI in order to avoid the thermal radiation from the vessel's walls.

TABLE II

MAIN OPTICAL SETUP SPECIFICATIONS

\begin{tabular}{|l|l|}
\hline Parameter & Value \\
\hline Wavelength Range & $0.8-2.5 \mu \mathrm{m}$ \\
QE Wavelength Range & $0.8-1.8 \mu \mathrm{m}$ \\
Bandwidth & $0.01 \mu \mathrm{m}$ \\
NEP @ QE Range & $<1.6 e^{-12} W^{1}$ \\
\hline
\end{tabular}

For quantum efficiency measures, an optical shortpass $(S P)$ filter with a custom cutoff wavelength of $1800 \mathrm{~nm}$ is used in order to avoid the thermal radiation produced by higher wavelengths and also for fitting with the NIST calibrated photodiode wavelength range used for irradiance calculation at the detector's position. Additionally, the cold baffle is wrapped with 10 sheets of multi-layer insulation $(M L I)$ in order to reject the thermal radiation coming from the vessel's walls, as shown in figure 9. Finally, the detector's focal plane is located at the end of the cold baffle, around $\sim 300 \mathrm{~mm}$ from the entrance window. The main specifications of the optical setup are described in Table II.

\section{CRYO-VACUUM SETUP DESCRIPTION}

The cryostat used in the setup has been fully designed and manufactured at IFAE and was previously used to perform the thermal cycles and further qualification and validation tests of the Filter Wheel Assembly $(F W A)$ of the ESA's Near Infrared Spectrograph and Photometer (NISP) instrument [23] [24] [25]. The vessel is made of aluminium with an inner volume of $\sim 120$ liters where

\footnotetext{
${ }^{1}$ Sampling Rate $=1$ sample $/$ second
}

a copper plate of $500 \times 500 \mathrm{~mm}$ is located, called cold plate, and allows to mount and align a custom optical system refrigerated up to $30 \mathrm{~K}$. Its huge cooling power is achieved thanks to a mechanically driven single-stage Gifford McMahon cold head Leybold COOLPOWER 250 $M D$ [26] with cooling capacity greater than $175 \mathrm{~W}$ at $80 \mathrm{~K}$, which is connected to a helium $(\mathrm{He})$ compressor unit Leybold COOLPACK 6000 HMD [26]. The vacuum is performed by a turbomolecular pump Agilent TV301 [27] which is connected to a dry scroll backing pump Agilent IDP-15 [27], both allowing to reach the last vacuum of $10^{-7}$ mbar at room temperature and $10^{-8} \mathrm{mbar}$ once the system is cold. The cold plate is made of a massive oxygen-free high thermal conductivity (OFHC) copper plate with an approximate weight of $\sim 20 \mathrm{Kg}$. It is thermally isolated from the vessel's walls by means of four PEEK ${ }^{\mathrm{TM}}$ spacers located at every corner and then attached to the cold head by a couple of copper straps sets. The detector is also mounted on a PEEK ${ }^{\mathrm{TM}}$ holder in order to isolate it from the whole assembly and is thermally connected to the cold plate by a thin copper braid.

Three heater elements of $100 \mathrm{~W}$ each have been installed to control the cold plate temperature. There are six PT-100 temperature sensors spread along the cold plate, the detector, the cold baffle and the radiation shield which are used for controlling and monitoring purposes. This way, there are two temperature closedloop controls (PID) which are performed by a Lakeshore 336 [28] controller: one to control the cold plate and the other to control the detector's temperature. The latter is performed by a built-in $15 \mathrm{~W}$ heater and its own calibrated Lakeshore PT-103 RTD sensor.

\section{A. CRYO-VACUUM SETUP CLEANLINESS}

The cryostat has been extensively tested to asses its reliability to host scientific-grade optical detectors. The vacuum cleanliness was investigated through the use of a MKS Microvison-IP [29] residual gas analyzer ( $R G A)$ which was connected to a $\mathrm{CF}$ port of the cryostat. This mass spectrometer measures the individual partial pressures of gases separating ions in the mean free path in the dewar's vacuum by their mass-to-charge ratio. The resulting atomic mass spectrum or cracking patterns, measured in atomic mass units $A M U$, is depicted in figure 10. It shows that the principal partial pressures belong to water (16, 17 and $18 \mathrm{AMU})$; atmospheric air gases: nitrogen (14 and $28 \mathrm{AMU})$, hydrogen (2 AMU), oxygen (32 AMU); some alcohol/acetone fragments (31 and 43 AMU) likely coming from the cleaning procedure and finally a small peak at 55 AMU which is hard to 


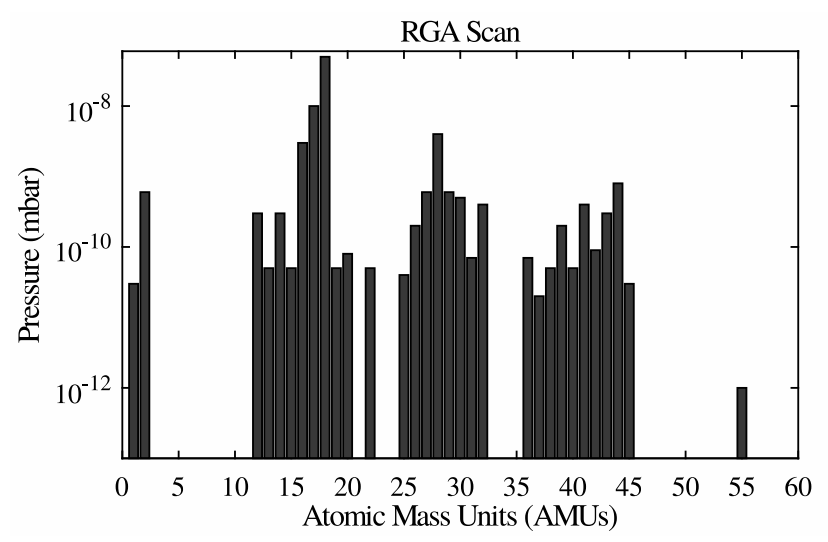

Fig. 10. Resulting atomic mass spectrum from the RGA during the cleanliness test. The highest peak belongs to water $(16,17$ and 18 AMU) and the lowest (55 AMU) probably to hydrocarbons.

classify but most probably coming from $\mathrm{C}_{4} \mathrm{H}_{7}^{+}$hydrocarbons [30]. Bearing all this in mind and regarding that the last reached vacuum is around $10^{-8}$ mbar, all the present gases have a melting point above $50 \mathrm{~K}$ and in the worse case of a hydrocarbon concentration, the peak is very low, we consider that the cryo-vacuum setup is safe to allocate high performance detectors without contaminating them.

\section{B. TEMPERATURE AND VACUUM CONTROL}

As described above, the temperature control of the cryo-vacuum setup is performed in two stages: one closed-loop control for the temperature of the cold plate and another for the detector's temperature. The control is performed by a Lakeshore 336 controller using its standard outputs: output 1 connected to the cold plate heater and output 2 to the detector's heater. Besides the temperature sensors used for control loop feedback, there are four sensors monitoring the temperature on critical parts which all together provide an overall view of the system performance.

The temperature error is a consequence of uncertainties on the temperature sensors and the electronics inaccuracies and is estimated to be around $16 \mathrm{mK}$ [31].

The whole cryo-vacuum system is controlled and monitored with LabVIEW ${ }^{\mathrm{TM}}$ [32] software through a state machine application, illustrated in figure 11, where every possible control configuration for the instruments is defined. Moreover, in order to perform the thermal cycles on the detector, the state machine takes decisions about the feasibility to cool down or warm up the detector after checking some basic safety conditions as the vacuum level, the turbomolecular pump state, the power line, etc. Once the safety condition is evaluated and a change in the state machine is approved, all

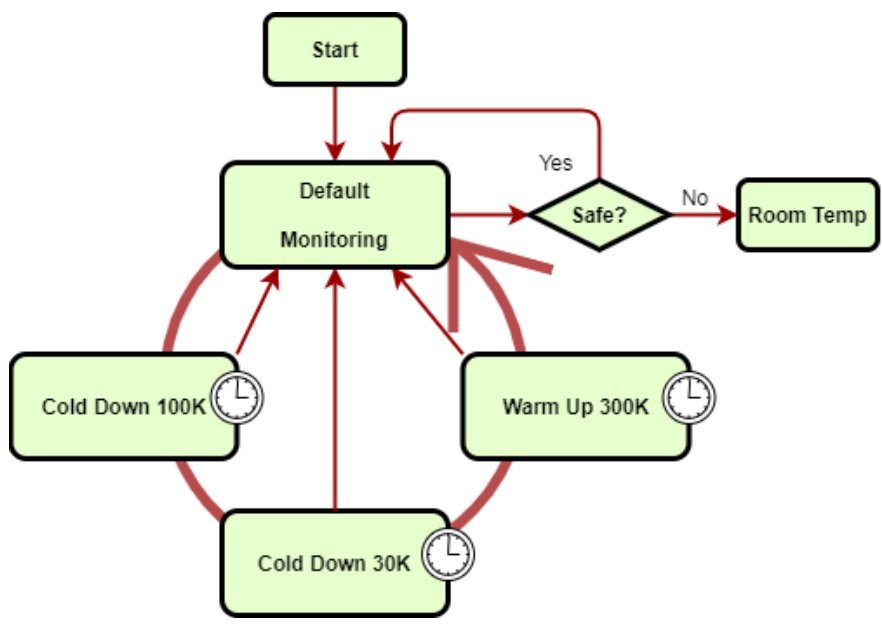

Fig. 11. State machine diagram of the cryostat control. The default state is monitoring while the data is logged and safety cross-checks are continuously performed.

the configuration parameters are sent to the different instruments; those parameters are mainly the temperature setpoints, the temperature and vacuum alarms thresholds. Following the instrument's configuration, the default state is monitoring, which $\log$ all the data in a text file and continuously evaluates the safety conditions associated to the current state.

The cold plate controllability is the key to guarantee the good performance of the whole thermal system since all the cold parts are thermally joined in such a piece: the cold optics, the cold baffle, the detector's package, the pre-amplification cage, etc. Due to the critical relevance of this system, the temperature stability, and thus its control, was specially investigated, simulated and tuned. After several iterations, the system has successfully achieved a temperature setpoint of $100 \mathrm{~K}$ with a controlled temperature ramp down of $0.5 \mathrm{~K} / \mathrm{min}$. A relative temperature error of $\sim 0.5 \mathrm{mK}$ was measured

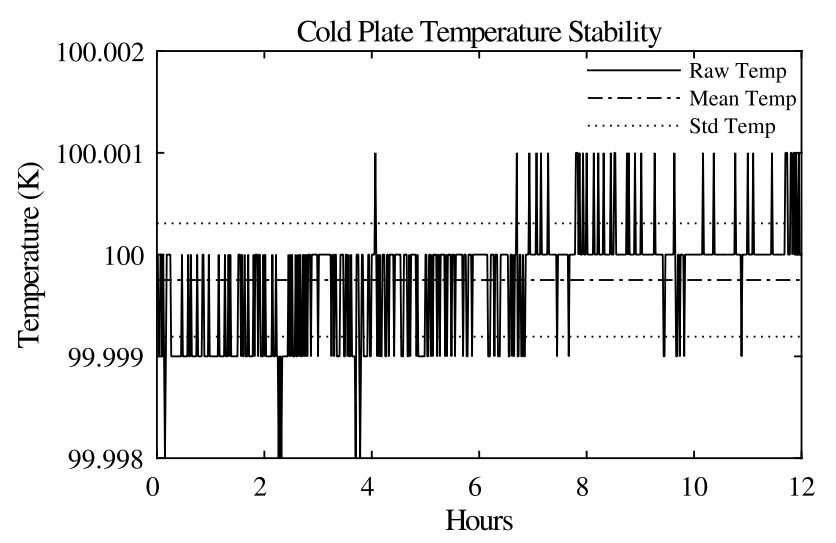

Fig. 12. Cold plate temperature stability in a 12-hour time-span with a relative temperature error of $\sim 0.5 \mathrm{mK}$ around the steady-state. 
along a 12-hour test, as can be seen in figure 12 .

\section{Conclusions}

Europe aims to be independent in manufacturing high performance and large focal plane arrays, being the ASTEROID detector the first european MCT-FPA device available for scientific use in the coming years. Detector characterization demands custom multidisciplinary setups with capabilities bordering the current engineering technologies, specially those developed for testing spaceborne scientific detectors, as is the case of the MCT focal plane arrays. The optical and the cryo-vacuum setups developed at IFAE were designed to measure the pixel operability while the detector is thermally stressed by repetitive thermal cycles but without being limited to perform the typical electro-optical battery tests used to report the detector performances. The setups allow to perform the optical performance detector tests in the range from 0.8 to $1.8 \mu \mathrm{m}$ with a $N E P$ $<1.6 e^{-12} \mathrm{~W} / \sqrt{(\mathrm{Hz})}$ while maintaining the detector at a temperature of $100 \mathrm{~K}$ with a temperature stability of $\sim 0.5 \mathrm{mK}$ and after performing the thermal cycle tests with a controlled ramp of $0.5 \mathrm{~K} / \mathrm{min}$. Thanks to the research done in this project and the achievements, it is demonstrated that the setups are commissioned and ready to install the ASTEROID detector for its further $E O$ characterization and thermal stress, offering homogeneous monochromatic illumination, high temperature stability and harmless vacuum environment.

\section{REFERENCES}

[1] O. Gravrand, J. Rothman, C. Cervera, N. Baier, C. Lobre, J. P. Zanatta, O. Boulade, V. Moreau, and B. Fieque. HgCdTe detectors for space and science imaging in France: general issues and latest achievements. In International Conference on Space Optics - ICSO 2016, volume 10562, pages 1177 - 1186. SPIE, 2017.

[2] O. Boulade, N. Baier, P. Castelein, C. Cervera, P. Chorier, G. Destefanis, B. Fièque, O. Gravrand, F. Guellec, V. Moreau, P. Mulet, F. Pinsard, and J.-P. Zanatta. Development and characterisation of MCT detectors for space astrophysics at CEA. In International Conference on Space Optics - ICSO 2014, volume 10563, pages 561 - 569. SPIE, 2017.

[3] Commissariat à l'énergie atomique et aux énergies alternatives (CEA)". [Online]. Available: http://www.cea.fr/. Accessed on: May, 2020.

[4] Lynred (Sofradir). [Online]. Available: https://www.lynred. com/. Accessed on: May, 2020.

[5] CEA-IRFU. [Online]. Available: http://irfu.cea.fr/en/. Accessed on: May, 2020.

[6] B. Fièque, A. Lamoure, F. Salvetti, S. Aufranc, O. Gravrand, G. Badano, O. Boulade, S. Mouzali, and S. Basa. Development of astronomy large focal plane array "ALFA" at Sofradir and CEA. In High Energy, Optical, and Infrared Detectors for Astronomy VIII, volume 10709, pages 33 - 51. SPIE, 2018.

[7] Asteroid Project webpage. [Online]. Available: https:// asteroidh2020.eu/. Accessed on: May, 2020.
[8] CEA-Leti. [Online]. Available: http://www.leti-cea.com/ cea-tech/leti/english. Accessed on: May, 2020.

[9] Institut de Física d'Altes Energies (IFAE). [Online]. Available: http://www.ifae.es/eng/. Accessed on: May, 2020.

[10] EV Group (EVG). [Online]. Available: https://www.evgroup. com/. Accessed on: May, 2020.

[11] ADDL. [Online]. Available: https://www.addl.fr/. Accessed on: May, 2020.

[12] P-E. Crouzet, J. ter Haar, F. de Wit, T. Beaufort, B. Butler, H. Smit, C. van der Luijt, and D. Martin. Test set up description and performances for HAWAII-2RG detector characterization at ESTEC. In High Energy, Optical, and Infrared Detectors for Astronomy $V$, volume 8453, pages 680 - 687. SPIE, 2012.

[13] NASA Detector Characterization Laboratory webpage. [Online]. Available: https://detectors.gsfc.nasa.gov/DCL/index.php. Accessed on: May, 2020.

[14] P.E. Crouzet. The new Teledyne NIR detectors for the SNAP/JDEM mission spectrograph. In Nuclear Instruments and Methods in Physics Research Section A: Accelerators, Spectrometers, Detectors and Associated Equipment, volume 610, pages 135 - 137, 2009.

[15] Gert Finger, et al. Performance evaluation and calibration issues of large format infrared hybrid active pixel sensors used for ground- and space-based astronomy. In Nuclear Instruments and Methods in Physics Research Section A: Accelerators, Spectrometers, Detectors and Associated Equipment, volume 565 , pages $241-250,2006$.

[16] Elizabeth H. Steenbergen, Christian P. Morath, Diana Maestas, Geoffrey D. Jenkins, and Julie V. Logan. Comparing II-VI and III-V infrared detectors for space applications. In Infrared Technology and Applications XLV, volume 11002, pages 299 307. SPIE, 2019.

[17] Antoni Rogalski. Infrared detectors: an overview. Infrared Physics \& Technology, 43(3):187 - 210, 2002.

[18] W. Tennant, D. Lee, and M. Zandian et al. Mbe hgcdte technology: A very general solution to ir detection, described by "rule 07", a very convenient heuristic. Journal of Electronic Materials, 37:1406-1410, 2008.

[19] Ian S. McLean. Electronic Imaging in Astronomy:Detectors and Instrumentation. Springer-Verlag Berlin Heidelberg, 2008.

[20] Jorge Jiménez, Otger Ballester, Laia Cardiel-Sas, Ricard Casas, Javier Castilla, Ferran Grañena, Juan de Vicente, Marino Maiorino, and Ignacio Sevilla. Test benches facilities for PAUCam: CCDs and filters characterization. In Ground-based and Airborne Instrumentation for Astronomy IV, volume 8446, pages 1962 - 1971. SPIE, 2012.

[21] Jorge Jiménez, José M. Illa, Juan de Vicente, and Ricard Casas. DESI-GFA testbench facilities for CCDs characterization. In Ground-based and Airborne Instrumentation for Astronomy VI, volume 9908, pages 2476 - 2482. SPIE, 2016.

[22] M. J. Persky. Review of black surfaces for space-borne infrared systems. In Review of Scientific Instruments, volume 70, pages 2193 - 2217, 1999.

[23] Thierry Maciaszek, et al. Euclid Near Infrared Spectrometer and Photometer instrument concept and first test results obtained for different breadboards models at the end of phase C. In Space Telescopes and Instrumentation 2016: Optical, Infrared, and Millimeter Wave, volume 9904, pages 298 - 315. SPIE, 2016.

[24] Giuseppe D. Racca, et al. The Euclid mission design. In Space Telescopes and Instrumentation 2016: Optical, Infrared, and Millimeter Wave, volume 9904, pages 235 - 257. SPIE, 2016.

[25] Euclid Project webpage. [Online]. Available: https://sci.esa.int/ web/euclid/. Accessed on: May, 2020.

[26] Leybold webpage. [Online]. Available: https://www.leybold. com/uk/en/. Accessed on: May, 2020. 
[27] Agilent webpage. [Online]. Available: https://www.leybold. com/uk/en/. Accessed on: May, 2020.

[28] Lakeshore webpage. [Online]. Available: https://www. lakeshore.com/. Accessed on: May, 2020.

[29] MKS webpage. [Online]. Available: https://www.mksinst.com/ c/mass-spectrometers/. Accessed on: May, 2020.

[30] ESO Outgassing of CCD Components in the Vacuum. [Online]. Available: https://www.eso.org/sci/facilities/develop/detectors/ optdet/docs/reports/contamination/msreport.html/. Accessed on: May, 2020.

[31] Appendix D: Sensor Calibration Accuracies Lakeshore. [Online]. Available: https://www.lakeshore.com/docs/ default-source/product-downloads/literature/lstc_appendixd_l. pdf/. Accessed on: May, 2020.

[32] Labview webpage. [Online]. Available: https://www.ni.com/ es-es/shop/labview.html/. Accessed on: May, 2020.

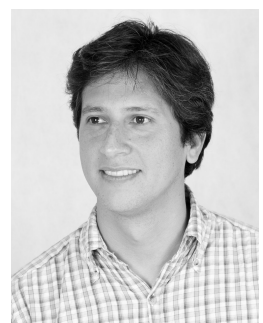

Jorge Jiménez received a B.E. degree in industrial engineering and a MSc. degree in control and robotics from the Technical University of Catalonia (UPC), Barcelona, Spain, where he is currently $\mathrm{PhD}$ candidate in the Control, Robotics and Vision program. He is detectors engineer at Institut de Física d'Altes Energies (IFAE) and during his career has been extensively working on instrumentation and detectors for astronomy. He early joined the instrumentation team at the ESO-VLT observatory (Atacama desert, Chile) where he participated in the maintenance, integration and upgrade of more than twelve cutting-edge technology instruments for the world's advanced optical observatory. At IFAE, he has lead the PAUCam camera CCDs characterization, a mosaic of eighteen CCDs already installed in the WHT telescope at the Canary Island in Spain, and characterized the CCDs used for the DESI-GFA, a novel guiding and focusing compactcamera developed at IFAE for the DESI instrument installed at the Mayall telescope in USA. He also collaborated with the University of Florida (USA) for the characterization of two infrared detectors for the MIRADAS instrument and currently, he is the ASTEROID Project Coordinator of the IFAE's workpackage.

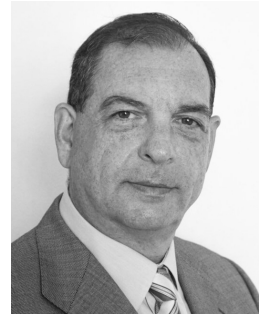

Antoni Grau received the M.S. and Ph.D. degrees in computer science from the Technical University of Catalonia (UPC), Barcelona, Spain, in 1990 and 1997, respectively. He is currently a Professor with the Department of Automatic Control, UPC, giving lectures on computer vision, digital signal processing, and robotics at the School of Informatics of Barcelona (FIB) and the College of Industrial Engineering of Barcelona (EEBE). He is also lecturer on Computer Vision at the Robotics and Vision Master at BarcelonaTech. Dr Grau is the Director of the "Control Engineering and Industrial Automation" postgraduate course. $\mathrm{He}$ is a co-author of a series of three books on programmable logic controllers and industrial communications. He also co-authored the book "Education for the Sustainability in Automatic Control". He is the author of more than 240 published papers. His research areas are computer vision, pattern recognition, robotics, factory automation, and education on sustainable development. Dr. Grau is member of the International Association for Pattern Recognition and the International Federation of Automatic Control. He chaired several international conferences. $\mathrm{He}$ is also member of IEEE Industrial Electronics Society (IEEEIES), co-chairing the Technical Subcommittee "FA11: Intelligent and Cooperative Robotics". He serves as Associated Editor of the Transactions on Industrial Informatics.

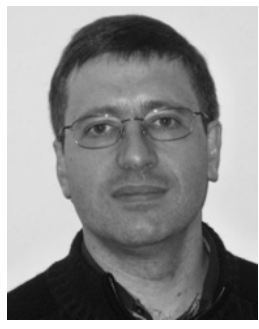

Cristobal Padilla made his $\mathrm{PhD}$ in Physical Sciences at the Universitat Autònoma de Barcelona (UAB) working for the LEP ALEPH experiment at CERN and has worked for many years in leading laboratories, like DESY (Hamburg, Germany) and CERN (Geneva, Switzerland). He has also made short visits to Los Alamos National Laboratory (USA), MPI-Heidelberg Karlsruhe Forschungszentrum (Germany), Paul Scherrer Insititut (Zurich, Switzerland), among others. He has been working in Particle Physics experiments for more than 25 years, where he has made substantial contributions in instrumentation, reconstruction and data acquisition software and analysis. For example, he has been Deputy Physics Coordinator at HERA-B, and responsible of the ATLAS High Level Trigger during the LHC start, a key system for the future discovery of the Higgs Boson. Since 2012 has made the transition to observational cosmology and is member of the PAU, DES, Euclid and DESC/LSST Collaborations, where he has made substantial contributions in instrumentation and data preparation. He has over 1000 publications with an h-index of 153 . He serves as editor of the Journal for Instrumentation and is currently the Director of the Technical Division of the Institut de Física d'Altes Energies (IFAE). 